\title{
High-dose-rate brachytherapy for high-grade vaginal intraepithelial neoplasia: a dosimetric analysis
}

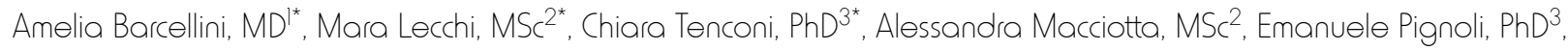 \\ Brigida Pappalardi, MD!, Ester Mazzarella, RTT³, Mauro Carrara, Phd³, Tommaso Giandini, PhD³, Carlo Fallai, MD', \\ Paolo Verderio, PhD², Annamaria Cerrotta, MD' \\ 'Radiotherapy 2 Unit, Fondazione IRCCS Istituto Nazionale dei Tumori, Milan, Italy, ${ }^{2}$ Bioinformatics and Biostatistics Unit, Fondazione IRCCS \\ Istituto Nazionale dei Tumori, Milan, Italy, ${ }^{3}$ Medical Physics Unit, Fondazione IRCCS Istituto Nazionale dei Tumori, Milan, Italy
}

*Amelia Barcellini, Mara Lecchi, and Chiara Tenconi contributed equally to this work.

\begin{abstract}
Purpose: Due to the rarity of vaginal intraepithelial neoplasia (VAIN), it is impossible to define the best treatment approach or to assess vaginal morbidity. However, brachytherapy (BT) could be a valuable choice for VAIN grade 3 (VAIN3). The aim of this paper was to report a single-institution study of the application of high-dose-rate BT and to evaluate clinical outcomes as well as to investigate the dose-effect relationship for vaginal stenosis.

Material and methods: We retrospectively collected hospital records and treatment plans of 14 consecutive women treated in our department from August 2010 to August 2016, with HDR-BT delivered using iridium-192 by a remote after-loading system. Doses in 3D-planned treatment based on computed tomography (CT) were prescribed in high-risk clinical target volume (HR-CTV) at the vaginal wall. Vaginal stenosis was defined as vaginal shortening/narrowing according to CTCAE4.1. The International Commission on Radiation Units \& Measurements (ICRU) bladder and rectal points were used for dose report analysis. The posterior-inferior border of the symphysis points was used to derive reference points. The median age of the enrolled women was 60 years, and the median total radiation dose delivered was 35 Gy.
\end{abstract}

Results: During a median period of 15 days, the treatment was well tolerated, and no interruption was necessary. Acute toxicity was minimal, whereas late toxicity appeared in 4 patients as G2 and in 3 patients as G3 vaginal stenosis. Patients with stenosis $G \geq 2$ received a higher median dose to the rectal point and were mainly over 60 years old.

Conclusions: Patients with VAIN3 seemed to benefit from BT. It is generally assumed that the vagina is radio-resistant, and no constraints have yet been set, but sexual dysfunction after BT is an important cause of long-term distress. Finding applicable dose limits to the vagina could improve patients' quality of life.

J Contemp Brachytherapy 2019; 11, 2: 146-151 DOI: https://doi.org/10.5114/jcb.2019.84696

Key words: vaginal intraepithelial neoplasia, vaginal toxicity, brachytherapy.

\section{Purpose}

Vaginal intraepithelial neoplasia (VAIN) is a rare condition, with $0.2-0.6$ cases per 100,000 women, and the number of cases seem to be increasing $[1,2,3]$. Taking into account all grades of untreated VAIN, $78 \%$ regress, $13 \%$ persist, and $9 \%$ progress to invasion [4]. With this low incidence, there is no certain treatment, although brachytherapy is a valid option. However, considering the quite long-life expectancy, long-term side effects due to radiation must be taken into consideration.
Although some vaginal morbidity as a result of radiation to a specific part of the vagina is to be expected, the literature offers little data about dose-effect relationships in the vagina. The physical changes related to radiation have been well described, including adhesion of vaginal walls, narrowing and shortening of the vaginal barrel, and loss of elasticity. A concomitant decrease in vaginal secretions leads to dyspareunia, with a significant impact on quality of life $[5,6,7,8,9]$.

We describe a single-institution retrospective study of the application of high-dose-rate (HDR) brachytherapy 
(BT) as a radical treatment or treatment at the time of the first relapse of VAIN grade 3 .

\section{Material and methods}

The records and treatment plans of 14 consecutive patients irradiated with HDR-BT between February 2010 and August 2016 for high-grade VAIN (grade 3) were analyzed. The following data were retrospectively collected for each case: patient demographics, clinical description of VAIN, previous treatments for VAIN, human papillomavirus (HPV) status, history of conization or hysterectomy, detailed description of BT, recurrence of VAIN or invasive vaginal carcinoma, and vital status.

HDR-BT was delivered using an iridium-192 ( $\left.{ }^{192} \mathrm{Ir}\right)$ source from Elekta Microselectron (Elekta, Stockholm, Sweden). The intracavitary vaginal BT technique at our institution is based on the single-channel vaginal cylinder or multichannel applicators. All HDR-BT plans were generated, with a 3D treatment planning system (Oncentra Brachy Nucletron Elekta, Veenedaal, The Netherlands) to calculate the dose on computed tomography (CT) and magnetic resonance (MR) images. Doses were prescribed in high-risk clinical target volume (HR-CTV) defined as vaginal wall ( $5 \mathrm{~mm}$ depth), with cervical surface if existing.

Patients were followed-up for six weeks after treatment, then every three months for the first two years, and twice per year thereafter. Vaginal smears were taken every six months, and vaginoscopies and biopsies were obtained whenever required to confirm a recurrence.

Late complications of the vagina were graded during follow-up examination using the Common Terminology Criteria for Adverse Events v.4.0 (CTCAE 4.0) [10]. We specifically considered vaginal stenosis, telangiectasia, and vaginal bleeding defined as follows:

- Vaginal stenosis: mild vaginal shortening or narrowing: asymptomatic (G1); not interfering with physical examination (G2) or interfering with the use of tampons, sexual activity, or physical examination (G3);

- Telangiectasia (TA): TA covering $<10 \%(\mathrm{G} 1)$ or $>10 \%$ (G2) of the vaginal mucosa;

- Vaginal bleeding: minimal bleeding identified on clinical examination and with no indication to intervention (G1); moderate bleeding, in which medical intervention was indicated (G2); severe bleeding, for which transfusion was indicated (G3).

Recurrent dysplasia or progression to invasive cancer was recorded from the last day of BT.

For the dose-report analysis, the International Commission on Radiation Units \& Measurements (ICRU) of bladder and rectal points [11] were used as surrogate points of the anterior and posterior vaginal wall, respectively. Both points were defined according to the patient coordinate system on anterior-posterior lines, which were strictly perpendicular to the patient's longitudinal axis. However, we did not use a classical tandem and ovoid or tandem and ring for treatment, the rectal point was positioned from the top of the vaginal cylinder on a straight anterior-posterior line and on the intersection on the axis perpendicular to the body axis, $5 \mathrm{~mm}$ dorsal to the posterior vaginal wall.
The vaginal applicators used meant that the posterior-inferior order of the symphysis (PIBS) vaginal dose points were not useful to study the dose along the vaginal axis [12]. PIBS points were used to derive reference points as surrogates for the dose distribution at the mid-vagina, the transition from mid to lower, and at the lower vagina as follows:

- Point 1 (P1): from the PIBS (defined as $2 \mathrm{~cm}$ posterior to the posterior-inferior border of the pubic symphysis) on a straight anterior-posterior line perpendicular to the patient axis at the point where this line crosses the urethra;

- Point 2 (P2): from P1, $2 \mathrm{~cm}$ up along the vaginal axis (at the level of PIBS+2);

- Point 3 (P3): from P1, $2 \mathrm{~cm}$ down along the vaginal axis (at the level of PIBS-2) (Figure 1).

$\mathrm{P} 1, \mathrm{P} 2$, and $\mathrm{P} 3$ were at a median distance of $0.5 \mathrm{~cm}$ (range, 0.4-0.6) from the vaginal applicators.

For each fraction, we retrospectively collected the dose received by these points. Total doses were recalculated to the biologically equivalent doses and fractionation given with 2 Gy per fraction (EQD2) using the equation: EQD2 $=$ nd $(\mathrm{d}+\alpha / \beta) /(2 \mathrm{~Gy}+\alpha / \beta)$, where $\mathrm{n}$ is the number of fractions and $d$ the dose (Gy) per fraction (assuming $\alpha / \beta=10$ Gy for tumor control, and $\alpha / \beta=3$ Gy for late normal tissue damage). For vaginal tissue, we assumed $\alpha / \beta=3$ because of the non-invasive nature of VAIN.

\section{Endpoints and statistical analysis}

The aim of this paper was to report a single-institution study of the application of high-dose-rate BT and to evaluate clinical outcomes as well as to investigate the dose-effect relationship for vaginal stenosis.

For the present analyses, we used information recorded in the dataset about age, total and fractional doses in Gy and EQD2 units, doses received by each point (P1, P2, P3, rectal and bladder, as specified above), type and corresponding grade of toxicity, previous treatment for VAIN (yes/no), and previous hysterectomy (yes/no). First, we did a descriptive statistical analysis, and data were presented with a boxplot, radar plot (continuous variables), or bar chart (discrete variables). Second, we used distribution-free tests (appropriate for small data sets and lack of knowledge of the underlying distribution), with an explorative aim, since they suffer low power. We used the

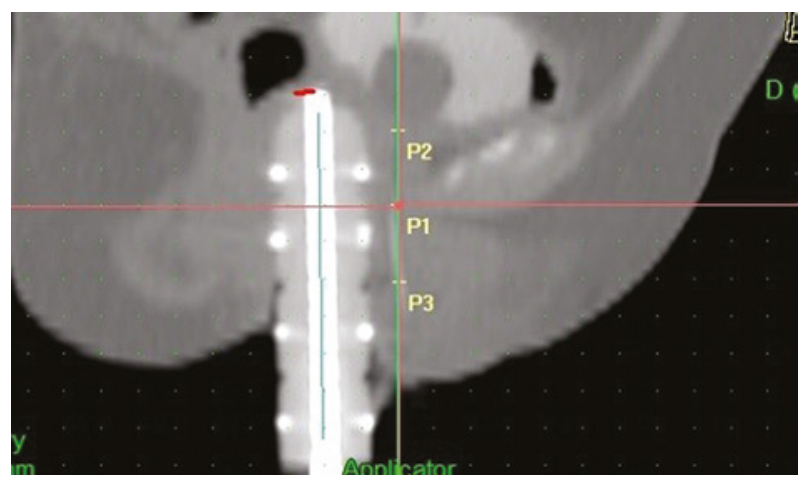

Fig. 1. Vaginal points 
Kruskal Wallis test and Fisher's exact tests to investigate the relations between continuous and categorical variables and vaginal stenosis $(G<2$ or $G \geq 2)$, respectively.

All statistical analyses were completed with the SAS (Version 9.4; SAS Institute, Inc., Cary, NC), adopting a significance level of $\alpha=0.05$.

\section{Results}

\section{Patients}

At diagnosis, all patients were asymptomatic, with HPV infection. Their ages ranged between 43 and 77 years (median, 60.5 years) and the median follow-up was 32.7 months (range, 10.5-89.83 months). Six patients had undergone hysterectomy for cervical cancer, three for cervical dysplasia, and one for benign disease. Two women had had previous treatment for VAIN grade 3 , one with 5-fluorouracil (5-FU) 5\% cream for vaginal self-application, and the other with laser ablation of the Lugol-identified vaginal lesion.

\section{Treatments}

All patients had plans for each fraction, and treatment was delivered twice a week for a mean of 15 days (range, 8-20 days). The total radiation dose range was 24-42 Gy, with a median of $35 \mathrm{~Gy}$; the total recalculated EQD2 range was 43.2-75.6 Gy (median, 56 Gy). Fraction sizes are reported in Table 1. Of the 14 registered patients, 13 had complete dosimetric data and were considered in the dosimetric analysis. Table 2 summarizes the doses received to P1, P2, P3, rectal, and bladder points.

Table 1. Treatment characteristics

\begin{tabular}{ll} 
Characteristics & Number of patients \\
\hline Fraction size & 7 \\
\hline 5 Gy $\times 7$ fractions & 1 \\
\hline 5 Gy $\times 6$ fractions & 1 \\
\hline $5.6 \times 7$ fractions & 1 \\
\hline $5.9 \times 7$ fractions & 1 \\
\hline 6 Gy $\times 4$ fractions & 3
\end{tabular}

After HDR-BT all patients used topical therapy with hyaluronic acid or/and vitamin E vaginal suppositories.

\section{Complications of brachytherapy}

Treatment was well tolerated, and no interruption was necessary. Acute toxicity was minimal, and most complaints were grade 1 urinary complications (5 patients). However, all patients had some late vaginal symptoms. During the follow-up, 5 patients developed vaginal stenosis G2 and 3 women had G3 (Figure 2). Since vaginal stenosis seemed to be the most frequent complaint, we focused our analysis by dichotomizing it on the basis of less than G2 or G2 and more.

Previous treatments for VAIN, previous hysterectomy, overall BT treatment time, total dose, and dose per fraction seemed not to be related to vaginal stenosis $G \geq 2$. Total doses delivered to each point are presented and according to the grade of stenosis in Figure3B. Per point dose and demographic analysis gave interesting (Figure $3 \mathrm{~A}$ ) results about the toxicity relationship with rectal point dose and age, respectively. Women with $G \geq 2$ stenosis received a higher median dose (Figures $3 \mathrm{C}$ and 4 ) and, in line with the published age cutoff, were $>60$ years old (Figure 5). No statistical significance was observed for these findings (Kruskal Wallis test: $p=0.391$, Fisher's exact test: $p=0.286$ for rectal dose and age analysis, respectively). However, the underpowered non-parametric test means it is hard to establish whether the real

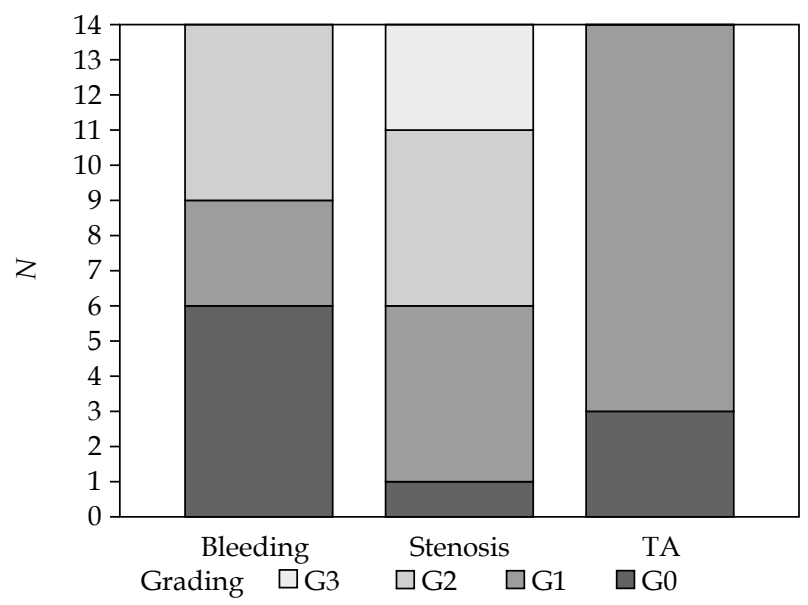

Fig. 2. Vaginal toxicity. Bar-chart showing the toxicity's grade of all patients, for each toxicity variable

Table 2. Descriptive statistics of dose (cGy) received to P1, P2, P3, rectal, and bladder points of the 13 analyzable patients

\begin{tabular}{lcccccc} 
Dose points & Minimum (cGy) & $2^{\text {th }}$ centile (cGy) & Median (cGy) & $7^{\text {th }}$ centile (cGy) & Maximum (cGy) & IQR (cGy) \\
\hline p1 & 1114.38 & 2702.44 & 3009.67 & 3163.01 & 4161.16 & 460.57 \\
\hline p2 & 2446.24 & 3042.46 & 3549.41 & 3742.42 & 4578.09 & 699.96 \\
\hline p3 & 404.05 & 1270.82 & 1576.30 & 1906.13 & 2492.77 & 635.31 \\
\hline Rectal & 2034.19 & 2801.51 & 3251.94 & 3978.17 & 5029.87 & 1176.66 \\
\hline Bladder & 1494.67 & 1851.20 & 2097.58 & 2426.68 & 2910.65 & 575.48
\end{tabular}

$I Q R$ - interquartile range, $75^{\text {th }}$ centile- $25^{\text {th }}$ centile 
A

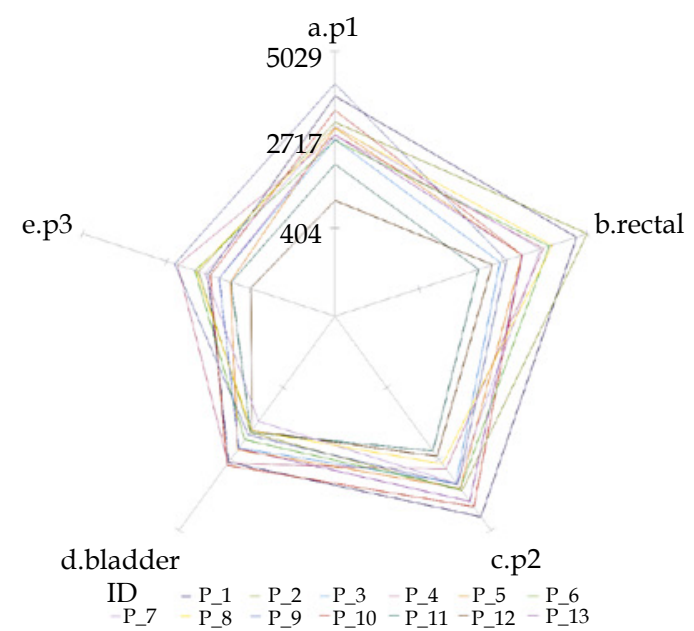

B

C

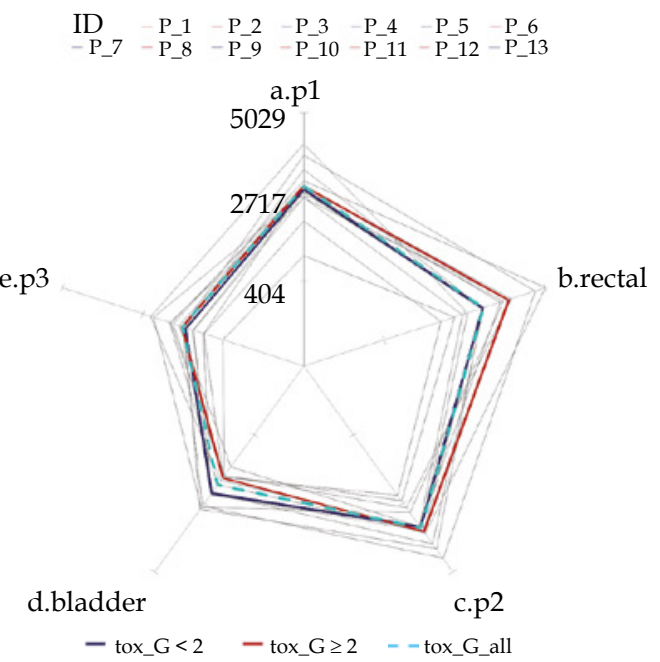

Fig. 3. Radar plots with radiation doses (cGy) depicted on different spokes, corresponding to P1, rectal, P2, bladder, and $\mathrm{P} 3$, in a clockwise direction. The distance between the point on the spoke and the center of the radar plot indicates the radiation dose in that PIBS-point. Lines connecting the points on different spokes give the dose pattern. A) Radar plot depicting the pattern of the radiation dose (cGy) for each patient. B) Radar plot depicting the pattern of the radiation dose (cGy) for patients with stenosis $G \geq 2$ (red lines) and $G<2$ (blue lines). C) Radar plot reporting the pattern of the median dose (cGy) calculated on the total of the patients (dotted line), on those with $G \geq 2$ (red line) and $G<2$ (blue line)

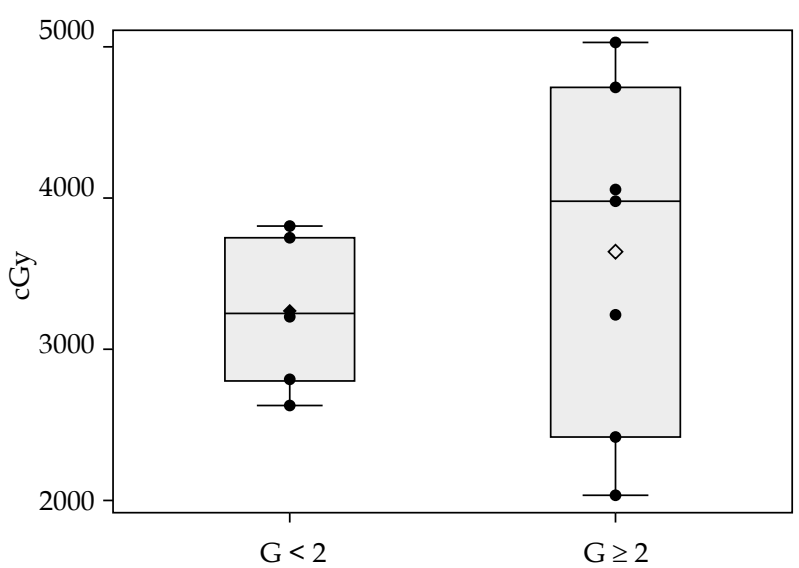

Fig. 4. Distribution of dose received to rectal point. Box plots reflecting the distribution of dose in patients with $G$ $<2$ and $G \geq 2$. Each box indicates the $25^{\text {th }}$ and $75^{\text {th }}$ centiles. The horizontal line inside the box indicates the median, and the whiskers indicate the extreme measured values. Each observation is represented by a dot

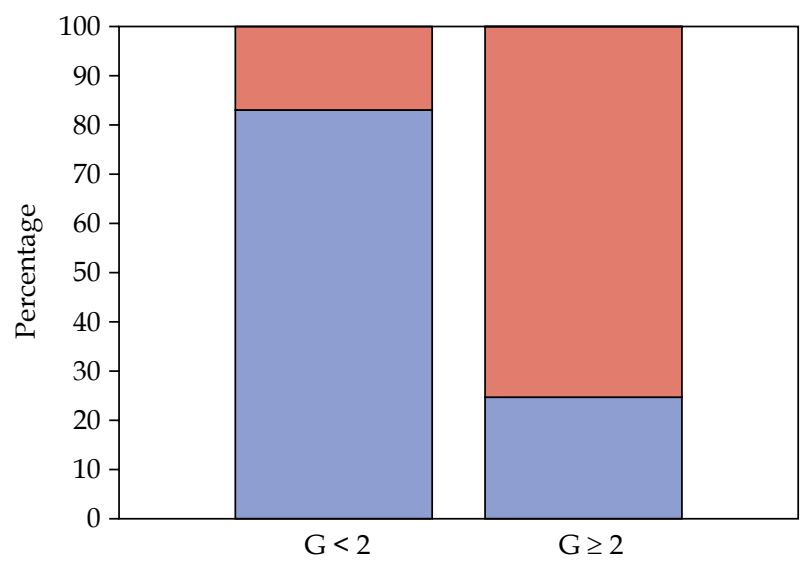

Fig. 5. Age and toxicity. Bar-chart showing the percentage of patients over (red) and under (blue) 60 years, in the two groups, based on the different grade of stenosis

differences are only too small to be detected or actually do not exist.

\section{Discussion}

Treatment for VAIN is based on grade and focality (uni- or multifocal), and the treatment modalities available are medical, surgical, and radio-therapeutic. For selected healthy women, conservative management may be considered. Medical management, which can give cure rates of $45-100 \%$ is recommended for patients with persistent HPV infection and multifocal VAIN. It consists of trichloroacetic acid, 5-fluorouracil, and an immune response modifier ( $5 \%$ imiquimod, cidofovir). Therapeutic vaccine expressed HPV 16-18 oncoproteins E6 and E7, and poly-gamma-glutamic acid is still under investigation $[13,14,15,16,17,18,19]$.

Surgical treatments involve $\mathrm{CO}_{2}$ laser ablation (successful in $70.8-79.2 \%$ ), surgical excision (successful in $66-87 \%$ ), cavitational ultrasonic surgical aspiration (CUSA), 
Table 3. Studies for vaginal intraepithelial neoplasia treated with brachytherapy

\begin{tabular}{|c|c|c|c|c|c|c|}
\hline Authors & $\begin{array}{l}\text { Number } \\
\text { of patients }\end{array}$ & Dose rate & Diagnosis & $\begin{array}{c}\text { Median fol- } \\
\text { low-up (months) }\end{array}$ & Total dose (Gy) & $\begin{array}{l}\text { Vaginal toxicity } \\
\text { (number of toxic- } \\
\text { ity/criteria) }\end{array}$ \\
\hline $\begin{array}{l}\text { Blanchard } \\
\text { et al. [28] }\end{array}$ & 28 & LDR & VAIN3 & 41 & 60 & $\begin{array}{c}\text { G1 (7) } \\
\text { (CTCAE) }\end{array}$ \\
\hline $\begin{array}{l}\text { Woodman } \\
\text { et al. [23] }\end{array}$ & 11 & LDR & VAIN3 & 25 & $27-51$ & $\begin{array}{c}\text { G2 (6) } \\
\text { G3 (1) } \\
\text { (EORTC/RTOG) } \\
\end{array}$ \\
\hline $\begin{array}{l}\text { Graham } \\
\text { et al. [27] }\end{array}$ & 22 & MDR & VAIN3 & 77 & 48 & $\begin{array}{c}\text { G3 (4) } \\
\text { G4 (1) } \\
\text { (EORTC/RTOG) }\end{array}$ \\
\hline $\begin{array}{l}\text { Song } \\
\text { et al. [29] }\end{array}$ & 34 & HDR & $\begin{array}{c}\text { VAIN } \\
\text { (all grade) }\end{array}$ & 48 & $30-50$ & $\begin{array}{c}\text { G1-2 (10) } \\
\text { G3 (2) } \\
\text { (EORTC/RTOG) }\end{array}$ \\
\hline
\end{tabular}

$L D R$ - low-dose-rate, MDR - medium-dose-rate, $H D R$ - high-dose-rate

with a cure rate of $74 \%[20,21,22]$. BT is acknowledged as an effective option, with high cure rates. In all reports, this treatment was well tolerated, with no limiting toxicities; the main late toxicity is vaginal, such as stenosis and ulcer (Table 3) [23,24,25,26,27,28,29].

After assessment by the multidisciplinary committee of gynecologic oncology, patients were selected for BT considering the multifocality of the disease. In our cohort, the HR-CTV is the whole length of the vagina because of the focality of VAIN. Since the vagina is also an organ at risk, we considered it worthwhile to assume $\alpha / \beta=3$ both for VAIN (a non-invasive disease) and for the whole vaginal tissue.

Vaginal BT may cause chronic vaginal alterations with direct damage to the vaginal mucosa, connective tissues, and microvasculature, resulting in secondary mucosal atrophy and increased collagen production that leads to shortening and tightening of the vagina. Pathological dilation of capillaries results in telangiectasia, tending to bleeding. In addition, adhesions and fibrosis cause loss of elasticity and some women may develop complete obliteration of vagina $[30,31,32]$.

Radiation-induced vaginal morbidity occurring within the first two years after the end of treatment is still a substantial problem, calling for further attention.

Since vaginal stenosis is the most frequent late effect and tends to persist, we focused our toxicity analysis on this issue. The damage to the epithelium was exacerbated by menopausal status, resulting in a further decrease in lubrication and thinning of vaginal tissues. Patients with stenosis more than or equal to 2 accounted for $86 \%$ and $29 \%$, respectively, among the over- 60 s and those aged 60 or less.

A points-based reporting method is generally used for the dose evaluation of bladder and rectum, but no clear recommendations for dose-volume parameters of the vagina have been developed so far. In the Embrace study (image-guided intensity-modulated external beam radiochemotherapy and MRI-based adaptive brachytherapy in locally advanced cervical cancer), the ICRU rectal point is suggested as a surrogate point to predict vaginal morbidity. Kirchheiner et al. showed an increase in the risk of vaginal stenosis for increasing the $\mathrm{EQD}_{2}$ dose to the reference recto-vaginal point, and a planning aim of 85 Gy EQD 2 (sum of external beam radiotherapy and BT) was proposed in order to reduce morbidity [33].

In our study, women who received a higher median dose to the rectal point developed major vaginal stenosis, even though we did not find any statistically significant evidence. Since non-parametric tests can suffer low power mainly due to small sample size, there may be a true effect or just a smaller difference that the underpowered non-parametric test cannot detect. For this reason, our results need to be reassessed in future studies.

Total dose and dose per fraction did not seem related to $G \geq 2$ vaginal stenosis. It is worth noting that one woman who received a total dose of 24 Gy in four fractions presented G3 toxicity after a follow-up of 37.53 months.

Major limitations of this study are the small sample and the retrospective analysis. However, the findings might prove useful as a starting point, since the rarity of this malignancy and limited BT approach for VAIN would require substantial resources for prospective studies.

\section{Conclusions}

This study found that HDR-BT was an effective, feasible, and well tolerated treatment for VAIN. In spite of high cure rates, we must still clarify issues regarding vaginal toxicity, the woman's age, and the total dose delivered to the rectal point in order to reduce complications.

Applicable dose limits to the vagina are needed to apply them when planning therapy to improve patients' quality of life. Therefore, this field merits further investigation to reach robust and significant results.

\section{Disclosure}

Authors report no conflict of interest.

\section{References}

1. Cramer DW, Cutler SJ. Incidence and histopathology of malignancies of the female genital organs in the United States. Am J Obstet Gynecol 1974; 118: 443-460.

2. Gemmell J, Holmes DM, Duncan ID. How frequently need vaginal smears be taken after hysterectomy for cervical intraepithelial neoplasia. Br J Obstet Gynaecol 1990; 97: 58-61. 
3. Arbyn M, de Sanjose S, Sarayia M et al. Eurogin 2011 roadmap on prevention and treatment of HPV-related disease. Int J Cancer 2012; 131: 1969-1982.

4. Gurumurthy M, Cruickshank ME. Management of vaginal intraepithelial neoplasia. J Low Genit Tract Dis 2012; 16: 306-312.

5. Grigsby PW, Russell A, Bruner D et al. Late injury of cancer therapy on the female reproductive tract. Int J Radiat Oncol Biol Phys 1995; 31: 1281-1299.

6. Mirabeau-Beale KL, Viswanathan AN. Quality of life (QOL) in women treated for gynecologic malignancies with RT: A literature review of patient-reported outcomes. Gynecol Oncol 2014; 134: 403-409.

7. Bergmark K, Avall-Lundqvist E, Dickman PW et al. Vaginal changes and sexuality in women with a history of cervical cancer. N Engl J Med 1999; 340: 1383-1389.

8. Brand AH, Bull CA, Cakir B. Vaginal stenosis in patients treated with radiotherapy for carcinoma of the cervix. Int J Gynecol Cancer 2006; 16: 288-293.

9. Bruner DW, Lanciano R, Keegan M et al. Vaginal stenosis and sexual function following intracavitary radiation for the treatment of cervical and endometrial carcinoma. Int J Radiat Oncol Biol Phys 1993; 27: 825-830.

10. Cancer Therapy Evaluation Program. Common Terminology Criteria for Adverse Events (CTCAE). Version 4.03.

11. International Commission on Radiation Units and Measurements (ICRU). Dose and volume specifications for reporting intracavitary therapy in gynecology. ICRU Report 38, Bethesda 1985.

12. Westerveld H, Pötter R, Berger D et al. Vaginal dose point reporting in cervical cancer patients treated with combined 2D/3D external beam radiotherapy and 2D/3D brachytherapy. Radiother Oncol 2013; 107: 99-105.

13. Lin H, Huang EY, Chang HY et al. Therapeutic effect of topical applications of tricholoroacetic acid for vaginal intraepithelial neoplasia after hysterectomy. Jpn J Clin Oncol 2005; 35: 651-654.

14. Haidopoulos D, Diakomanolis E, Rodolakis A et al. Can local application of imiquimod cream be an alternative mode of therapy for patients with high-grade intraepithelial lesions of the vagina? Int J Gynecol Cancer 2005; 15: 898-902.

15. Diaz-Arrastia C, Arany I, Robazetti SC et al. Clinical and molecular responses in high-grade intraepithelial neoplasia treated with topical imiquimod 5\%. Clin Cancer Res 2001; 7: 3031-3033.

16. Buck HW, Guth KJ. Treatment of vaginal intraepithelial neoplasia (primarily low grade) with Imiquimod 5\% cream. J Low Genit Tract Dis 2003; 4: 290-293.

17. Tristam A, Fiander A. Clinical responses to cidofovir applied topically to women with high grade vulval intraepithelial neoplasia. Gynecol Oncol 2005; 99: 652-655.

18. Van Pachterbeke C, Bucella D, Rozenberg S et al. Topical treatment of CIN2+ by cidofovir : results of a phase II, double-blind, prospective, placebo-controlled study. Gynecol Oncol 2009; 115: 69-74.

19. Baldwin PJ, van der Burg SJ, Boswell CM et al. Vaccinia-expressed human papillomavirus 16 and 18 E6 an E7 as therapeutic vaccination for vulval and vaginal intraepithelial neoplasia. Clin Cancer Res 2003; 9: 5205-5213.

20. Koo YJ, Min KJ, Hong JH et al. Efficacy of polygamma- glutamic acid in women with high-risk human papillomavirus-positive vaginal intraepithelial neoplasia: an observational pilot study. J Microbiol Biotechnol 2015; 25: 1163-1169.

21. Yalcin OT, Rutherford TJ, Chambers SK et al. Vaginal intraepithelial neoplasia: treatment by carbon dioxide laser and risk factors of failure. Eur J Obstet Gynecol Reprod Biol 2003; 106: 64-68.
22. von Gruenigen VE, Gibbons HE, Gibbins K et al. Surgical treatments for vulvar and vaginal dysplasia, a randomized controlled trial. Obstet Gynecol 2007; 109: 942-947.

23. Woodman CB, Mould JJ, Jordan JA. Radiotherapy in the management of vaginal intraepithelial neoplasia after hysterectomy. Br J Obstet Gynaecol 1988; 95: 976-979.

24. MacLeod C, Fowler A, Dalrymple C et al. High-dose-rate brachytherapy in the management of high-grade intraepithelial neoplasia of the vagina. Gynecol Oncol 1997; 65: 74-77.

25. Ogino I, Kitamura T, Okajima $\mathrm{H}$ et al. High-dose-rate intracavitary brachytherapy in the management of cervical and vaginal intraepithelial neoplasia. Int J Radiat Oncol Biol Phys 1998; 40: 881-887.

26. Teruya Y, Sakumoto K, Moromizato H et al. High dose-rate intracavitary brachytherapy for carcinoma in situ of the vagina occurring after hysterecto-my: a rational prescription of radiation dose. Am J Obstet Gynecol 2002; 187: 360-364.

27. Graham K, Wright K, Cadwallader B et al. 20-year retrospective review of medium dose rate intracavitary brachytherapy in VAIN3. Gynecol Oncol 2007; 106: 105-111.

28. Blanchard P, Monnier L, Dumas I et al. Low-dose-rate definitive brachytherapy for high-grade vaginal intraepithelial neoplasia. Oncologist 2011; 16: 182-188.

29. Song JH, Lee JH, Lee JH et al. High-dose-rate brachytherapy for the treatment of vaginal intraepithelial neoplasia. Cancer Res Treat 2014; 46: 74-80.

30. Kirchheiner K, Fidarova E, Nout RA et al. Radiation induced morphological changes. Strahlenther Onkol 2012; 188: 1010-1019.

31. Kirchheiner K, Nout RA, Tanderup K et al. Manifestation pattern of early-late vaginal morbidity after definitive radiation (chemo)therapy and image-guided adaptive brachytherapy for locally advanced cervical cancer: an analysis from the EMBRACE study. Int J Radiat Oncol Biol Phys 2014; 89: 88-95.

32. Morris L, Do V, Chard J et al. Radiation-induced vaginal stenosis: current perspectives. Int J Womens Health 2017; 9: 273-279.

33. Kirchheiner K, Nout RA, Lindegaard JC et al. Dose-effect relationship and risk factors for vaginal stenosis after definitive radio(chemo)therapy with image-guided brachytherapy for locally advanced cervical cancer in the EMBRACE study. $R a$ diother Oncol 2016; 118: 160-166. 